\title{
Double-blind, randomized, placebo controlled trial on the effect of 10 days low-frequency rTMS over the vertex on sleep in Parkinson's disease
}

\author{
Pablo Arias ${ }^{\text {a }}$, Jamile Vivas ${ }^{\text {a }}$, Kenneth L. Grieve ${ }^{\text {a,b }}$, Javier Cudeiro ${ }^{\text {a }}$ \\ ${ }^{a}$ Neuroscience and Motor Control Group (NEUROcom), Department of Medicine-INEF and Institute for Biomedical Research \\ (INIBIC), University of A Coruña, Spain \\ ${ }^{b}$ Faculty of Life Sciences, The University of Manchester, UK
}

\begin{abstract}
Objective: A recent report indicates repetitive transcranial magnetic stimulation (rTMS) improves sleep in Parkinson's disease (PD). The aim of this work is to evaluate the effect of 10 days rTMS on sleep parameters in PD patients.

Methods: Double-blind, placebo-controlled design. Eighteen idiopathic PD patients completed the study. Sleep parameters were evaluated through actigraphy and the Parkinson's Disease Sleep Scale (PDSS), along with depression (Hamilton Depression Rating Scale, HDS), and the Unified Parkinson's Disease Rating Scale (UPDRS). Evaluations were carried out before treatment with rTMS (pre-evaluation, PRE), after the rTMS treatment programme (post-evaluation, POST), and one week after POST (POST-2). Nine PD patients received real rTMS and the other 9 received sham rTMS daily for 10 days, (100 pulses at 1 $\mathrm{Hz}$ ) applied with a large circular coil over the vertex.

Results: Stimulation had no effect over actigraphic variables. Conversely PDSS, HDS, and UPDRS were significantly improved by the stimulation. Notably, however, these changes were found equally in groups receiving real or sham stimulation.

Conclusions: rTMS, using our protocol, has no therapeutic value on the sleep of PD patients, when compared to appropriate sham controls. Future works assessing the possible therapeutic role of rTMS on sleep in PD should control the effect of placebo.
\end{abstract}

Keywords: Parkinson. Sleep. Repetitive transcranial magnetic stimulation (rTMS). Actigraphy. Depression. Therapy

\section{Introduction}

Parkinson's disease (PD) is almost invariably thought of as a disease of the motor system [1]. However, it is well documented that PD displays a whole range of non-motor signs, amongst which sleep disorders affect 74-98\% of patients [2,3], though it seems all patients display alterations in the early phases of the disease [4]. It has been suggested that the course of such disturbances might be not directly related to the motor progression of the disease [5]. Given that sleep disorders (along with depression and loss of independence) are factors with the highest impact in quality of life of patients [6], investigation into the nature of this phenomenon is important. Sleep disorders in PD are well defined and can be subdivided into daytime and nocturnal occurrences [2,7]. Amongst the latter, light fragmented sleep is the most frequent subjective sleep complaint in PD [8]. Sleep disorders are associated with other non-motor symptoms of the disease, as there is a strong relationship between subjective sleep complaints and depression, particularly in PD [8].

The origin of sleep disorders in PD could be the result of side-effects of medication, as dopaminergic agonist has been shown to worsen sleep efficiency [9]. Secondly, there may be neurodegeneration of sleep regulating structures during the progression of the disease [2,7]. It also seems possible that the sleep disorders relate to dopaminergic function in parallel with non-dopaminergic dysfunction [10], e.g., light fragmentation sleep has been improved by dopaminergic treatment [11]; though other studies have reported sleep disorders only partially respond to dopaminergic treatment, as controlled released levodopa increases sleep time but has no effect on sleep fragmentation [12]. In contrast to motor symptoms for 
which several treatments are available, sleep disorders in PD are usually poorly recognized and treated [4].

Transcranial magnetic stimulation (TMS) is a technique useful for evaluating several neurophysiological parameters [13] and potentially treating non-motor [14] and motor disorders [15]. The therapeutic role of repetitive TMS (rTMS) is based on its ability to differentially modulate neural activity depending on stimulation parameters such as frequency [16], coil type [17], pulse waveform [18,19], or current direction [20]. This may be the reason that a single recent study (published whilst our work was in process) has shown beneficial changes to sleep following rTMS in PD [21]. The study showed that 10 days of $5 \mathrm{~Hz}$ rTMS over the parietal cortex improved sleep efficiency and fragmentation index as assessed by actigraphy. These results are intriguing as they seem to suggest a possible therapeutic role for rTMS in improving sleep quality in PD, with the group receiving parietal stimulation compared to a control group receiving motor cortex stimulation. Interestingly, it has been reported that rTMS over the motor cortex induces an increase in slow wave activity (SWA) in subsequent sleep in a group of healthy subjects [22] so that given the small proportion of SWA in PD [23] the use of rTMS to improve sleep in $\mathrm{PD}$ is worth being explored. It has also been shown (again in healthy subjects [24]) that low-frequency rTMS increases SWA.

Taking all the above into account, we decided to study the effect of 10 days low-frequency $(1 \mathrm{~Hz})$ rTMS applied over the vertex, previously used with apparent success on motor signs [25,26], using a diffuse large round coil applied on sleep (and depression) in PD, using a double-blind, placebo-controlled design. We hypothesized that rTMS, under the protocol tested, would have a positive impact on sleep disorders in PD.

\section{Methods}

\subsection{Patients}

Nineteen PD patients started and 18 PD (9 real, 9 sham) patients completed the study. Inclusion criteria were primary diagnosis of Parkinson's disease (based on medical records following established diagnostic criteria). Exclusion criteria were previous experience with TMS; implanted devices; pumpbased drug administration systems; history of seizures; dementia (MMSE <24); presence of dyskinesias disturbing stimulation; unpredictable motor fluctuations; and medication change within the last month before the start of experiment. One of the 19 PD patients who started the study withdrew for reasons unconnected to the study. The rest completed the protocol without complaint. Patients taking drugs to assist sleep or antidepressant were not included in the study (Fig. 1).

Patients were required to complete all sessions and could not change their regime of medication throughout the study. Patients were enrolled into the programme in groups of up to 4 patients every 3 weeks. Patients were randomly assigned to either receive real or sham stimulation during the sessions; however, the distribution of the two last sets of patients followed a counterbalance design (based on PRE UPDRS-score) in order to avoid an imbalance in the groups. The selection of UPDRS as criterion was based on previous reports showing that sleep disorders do not match motor progression of the disease [5].

\subsection{General procedure}

Evaluations were carried out three times: pre-test, before the 10 stimulation sessions (PRE); post-test, after the stimulation programme (POST); and one week after POST (POST-2). Evaluation was performed by investigators blind to the whole design and was performed during patient ON periods as defined by neurological examination and patient's confirmation. TMS (Monday-Friday for two weeks) was applied in the morning during patient-rated ON periods, between 1 and $2 \mathrm{~h}$ after medication intake, based on previous work reporting better effect of stimulation when applied during ON periods [27].

The protocol conformed to the Helsinki declaration and was approved by the ethics committee of the University of A Coruña. All patients were fully informed and signed consent forms.

\subsection{Evaluations}

\subsubsection{Actigraphy}

We used the Actiwatch system (Actiwatch, CamNtech Ltd., Cambridge, UK) to register sleep parameters. Actigraphy is recognized as a useful tool for the assessment of sleep quality [28], which has been favourably compared to polysomnography [29]. It has also been shown to be suitable for assessing sleep quality in PD [9,30]. 
Patients wore the system, based on accelerometers built into wrist-worn activity watches which were attached to the wrist of the least affected side. Patients wore the system for a period of three days and three nights immediately before (PRE) and after the stimulation period (POST) and one week after POST (POST-2). Patients wore the watch all day, except during bathing. Patients were instructed to press the Actiwatch button when turning lights off and again when getting out of bed; a "sleep diary" was kept reflecting the times of getting into and out of bed, with other complementary data such as the number of times they got up during the night, etc.

\section{Trial Flow-chart}

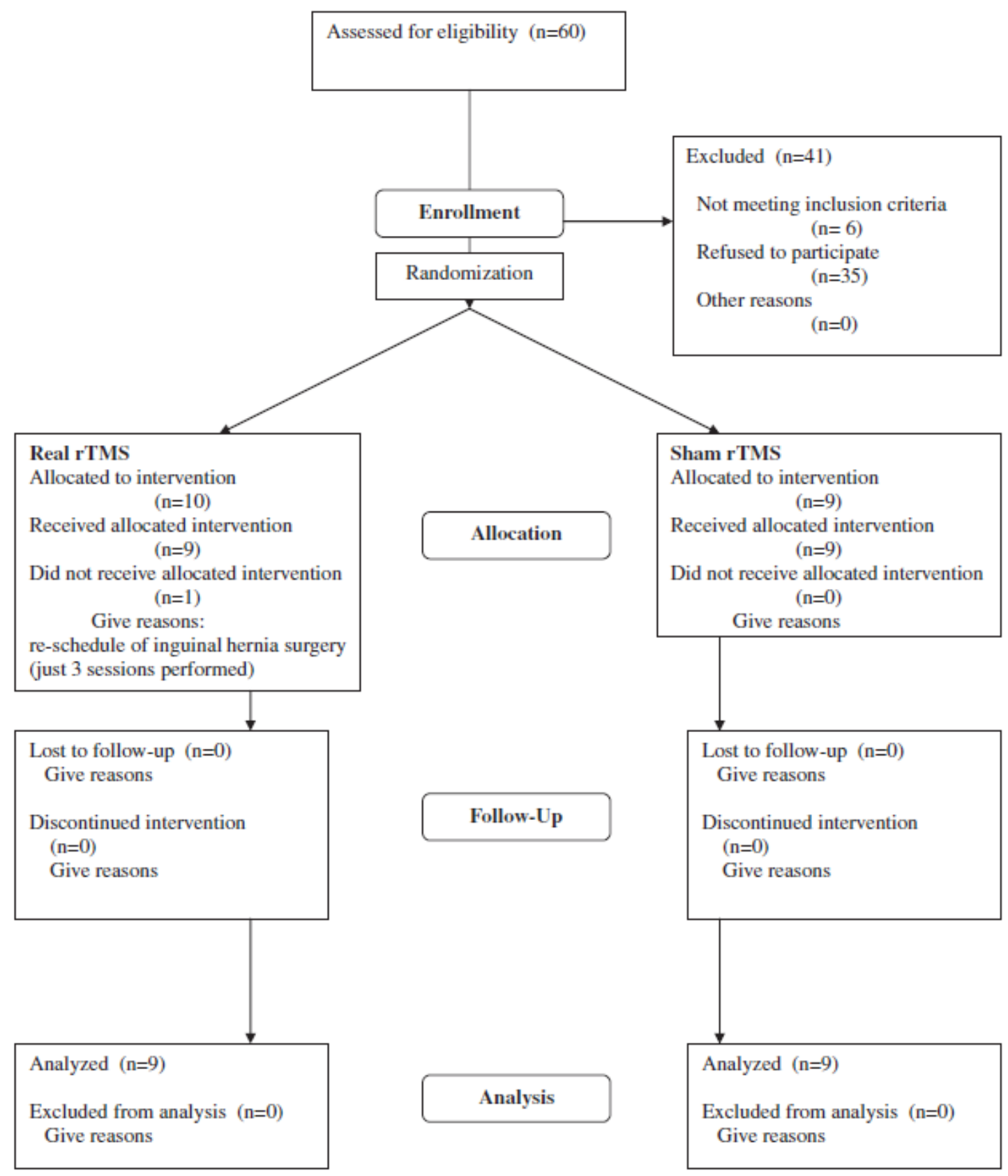

Fig. 1. Trial flow-chart. 


\subsubsection{Parkinson's Disease Sleep Scale (PDSS)}

The PDSS is a reliable scale for the assessment of subjective sleep complaints, specifically developed for PD [31,32]. This has been validated in its Spanish version [33] and successfully compared to other sleep assessment techniques [34]. The scale covers several sleep disorders related to the nocturnal and daytime manifestations of the disease. These are assessed by 15 items to be addressed using an analogue scale, toward a total score ranging from 0 to 150: the lower the score, the greater the sleep complaints. Patients were asked to complete the scale three times (at PRE, POST, and POST-2), in each case concerning the week preceding the test period.

\subsubsection{Hamilton Depression Scale (HDS)}

The HDS-17 [35], which has also been validated in Spanish [36], was used in order to assess depression. Evaluation again referred to the week preceding the test period.

\subsubsection{UPDRS}

The total score of the UPDRS was assessed.

\subsection{Stimulation}

TMS was applied using a round coil (see Apparatus) centred over the vertex. For each session, 100 pulses were delivered in two trains of 50 pulses/train, applied at $1 \mathrm{~Hz}$. One train was delivered clockwise and the other one anti-clockwise. We allowed a 5 min pause between trains. Intensity was set at $90 \%$ of the rest motor threshold (RMT), which was calculated every day before starting the stimulation. RMT was defined as the lowest intensity required to elicit a clear response of $50 \mu \mathrm{V}$ peak-to-peak amplitude in 5 out of 10 successive trials in the first dorsal interosseus of the less affected side.

For sham stimulation two coils were used. One coil was not connected to the stimulator, and this coil was placed over the vertex, as the real coil would have been. The second coil was placed in direct contact to the first coil, but tilted $90^{\circ}$. In this way the patient could feel a coil flat over the head (as was expected from the description given to them prior to the test), but be unaware of the presence of a second, active coil (but able to appreciate the sensory input associated with the presence of a "coil"). During the sham protocol EMG settings were maintained and some single pulses were delivered (mimicking RMT determination) in order to make protocols appear identical.

Therefore, the amount of time delivering stimulation per sessions in our protocol lasted the same as in a study by van Dijk et al. [21], since we both stimulated during $100 \mathrm{~s} /$ session; though given that our stimulation frequency was $1 \mathrm{~Hz}$, following previous findings [24], stimulation blocks were different.

\subsection{Apparatus}

Transcranial magnetic stimulation: TMS stimulation was applied using a High Power $90 \mathrm{~mm}$ Round Coil powered by a Magstim Rapid device (The Magstim Company, Whiyland, UK). Motor evoked potentials (MEP) were registered using $\mathrm{Ag} / \mathrm{Cl}$ surface electrodes fixed on the skin with a belly tendon montage.

Actiwatch system: actigraphy recording is based on registration movement by the means of a piezoelectric accelerometer (built into the wrist-watch-like device) that is designed to record the integral of intensity, amount and duration of movement in all directions. Activity is then converted to a measured in counts. Determination of sleep and wakefulness by the Actiwatch Sleep Analysis software relies on an algorithm which uses the activity data recorded by the device in a series of linked calculations. The algorithm looks at each data point from each epoch and those surrounding it and makes a total score based on these activity counts.

\subsection{Analysed variables}

The following variables were analysed in this study:

Actigraphic variables (all their units are defined in the tables)

- Immobility phases of $1 \mathrm{~min}$ (total and\%): the number of immobility phases where the duration was only one minute; its percentage represents the proportion of immobility phases of $1 \mathrm{~min}$ with regard to the total number of immobility phases. 
- Number of sleep and wake bouts: the actual number of episodes of sleep and wakefulness

- Mean length of sleep and wake bouts: total duration of sleep and wake by the corresponding number of sleep or wake bouts.

- Mean length of immobility: the average length of all the immobile phases during the time in bed.

- Number of immobile phases (total and\%): the number of periods of continuous scores of zero being recorded in consecutive epochs.

- Number of minutes immobile (total and\%): the total number of minutes where a score of zero was recorded during the assumed sleep period; its percentage represents the percentage of time spent immobile during assumed sleep period.

- Number of minutes moving (total and\%): the total number of minutes where a score greater than zero was recorded during the assumed sleep period; its percentage represents the percentage of time spent moving during assumed sleep period.

- Sleep latency: latency before sleep onset following bed-time.

- Sleep efficiency: percentage of time spent asleep whilst in bed.

- Movement and fragmentation index: the addition of percentage time spent moving and the percentage immobility phases of $1 \mathrm{~min}$. It is an indicator of restlessness.

PDSS, HDS, UPDRS: scores from each scale were introduced into the analysis.

\subsection{Data analysis}

To check homogeneity between groups before the start the stimulation protocol (at PRE), a Students " " $t$ " for independent samples, was applied for each variable.

Afterwards, to evaluate the effect of the stimulation, a two-way ANOVA with repeated measures was performed for each of the variables analysed. One within-subjects factor was defined, factor evaluation with 3 levels (PRE, POST, POST-2); this would therefore measure the effect of the protocol on the variables. The between subjects factor was the group (real stimulation or sham stimulation), which therefore was set to measure a possible placebo effect.

During analysis the normality of distribution was checked by a K-S test. In the ANOVA analysis the degrees of freedom were corrected by applying the Greenhouse-Geisser correction when sphericity was violated. The SPSS package was used for statistical analysis and significance was set at $p<0.05$.

\section{Results}

\subsection{Groups homogeneity. Comparison at PRE values}

Groups were not significantly different for all variables at the beginning of the protocol. When the different variables were compared between the groups receiving real or sham stimulation, before starting the protocol, Student " $t$ " for independent samples did not show any significant difference (see Table 1).

\subsection{Effect of the stimulation protocol}

The result of the rTMS protocol on the variables had two welldefined patterns of effects. Using the analysis rituals described, the UPDRS scores, HDS and PDSS were significantly improved following treatment $(F(2,32)=13.077 p \leq 0.001, F(2,32)=23.508 p \leq 0.001$ and $F(1.412,22.591)=7.902 p=$ 0.005 , respectively), indicating that the treatment produced benefits across the whole population. In addition, the interaction Evaluation * Group showed no significant differences [for the measures UPDRS, HDS and PDSS; $F(2,32)=0.704 p=0.502, F(2,32)=0.389 p=0.681$ and $F(1.412,22.591)=2.932 p=$ 0.089 , respectively; see Table 2], indicating a significant placebo effect.

In addition, it is notable that no actigraphic variable was affected by the stimulation. This pattern was seen in both groups (as there was no significant interaction in Evaluation * Group). Therefore, it is clear that the rTMS protocol had no effect on these parameters (see Table 3). Also factor group for all the analysed variables was not significant either (see Tables 2 and 3). 
Table 1

Characterization of groups before starting the stimulation protocol.

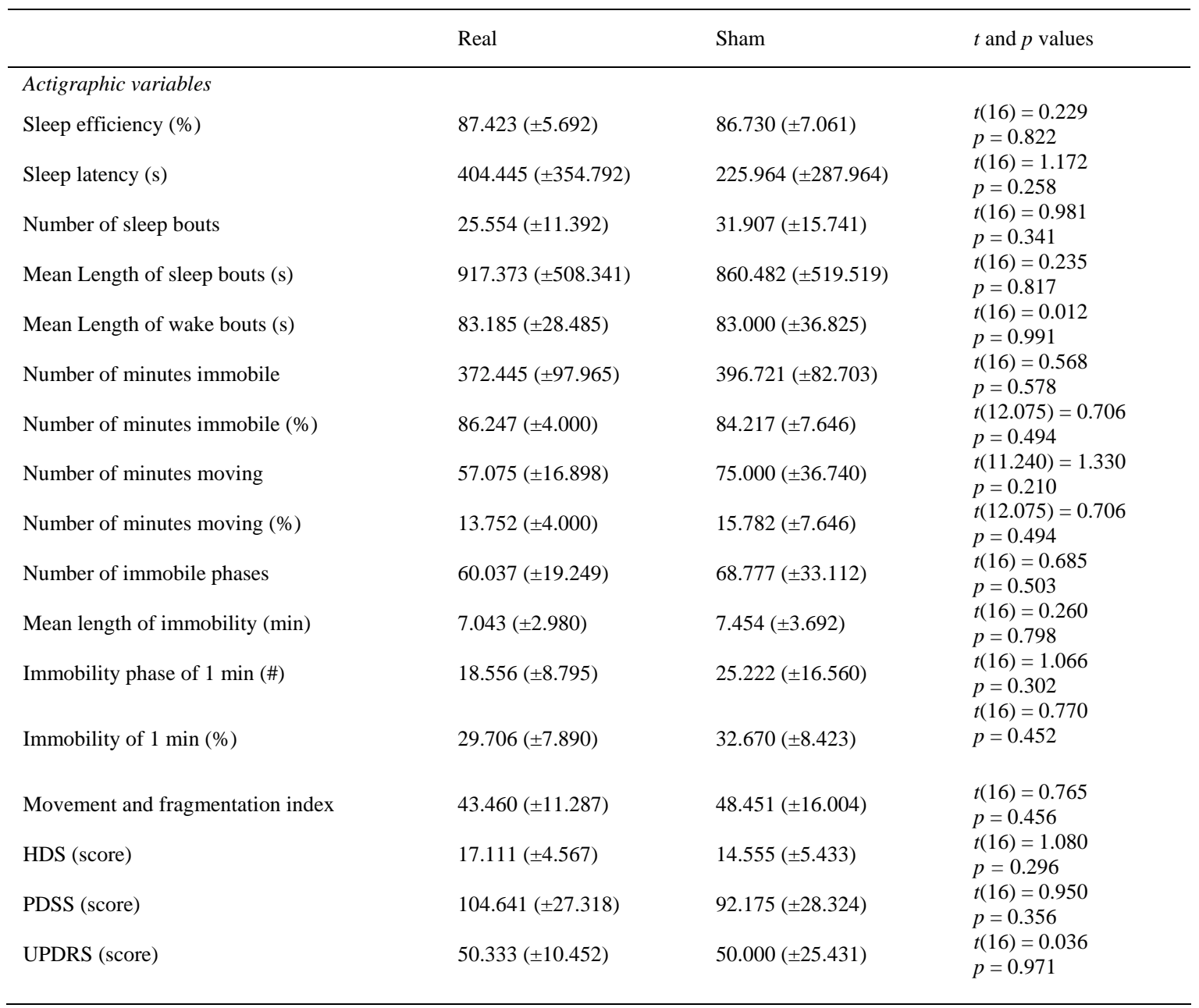

The table represents the value for each variable before starting the protocol. Lack of significant differences (real vs. sham) at the pre-test values means groups was comparable and homogeneous at the beginning of the programme. Means and standard deviations are displayed along with the p value.

Abbreviations: HDS, Hamilton Depression Scale; PDSS, Parkinson's Disease Sleep Scale; UPDRS, Unified Parkinson's Disease Rating Scale.

\section{Discussion}

The main outcome of the study is that the protocol used has no therapeutic value as a therapy for sleep disorders in PD. Our results did not show any change in the sleep parameters objectively evaluated. The data does, however, display significant changes in the PDSS (a well designed scale used to assess subjective sleep complaints in PD) and in the score for depression and the UPDRS, all of which were positively affected. But in each of these cases improvement seems clearly attributable to a placebo response. The results from PDSS are complementary to those measured by actigraphy, as PDSS covers Parkinsonian-related sleep complaints displayed also during daytime; this reinforces the idea of a placebo response cause of improvements which do not appear in the "sleeping" patient. Interestingly, our data showed a significant improvement on measures of depression, also attributable to placebo response.

The traditional view of Parkinson's disease is based firmly upon the (more obvious) motor signs, and treatments have invariably been directed towards the goals of amelioration (quite rightly) of these major crippling symptoms. However, non-motor symptoms exist in Parkinsonian syndrome, which are now receiving more attention; amongst these, depression and sleep disorders significantly lower the sufferer's quality of life. To our knowledge only one recent study [21] has reported the effect of rTMS on sleep parameters, which included an improvement in two out of seven actigraphic variables, fragmentation 
Table 2

Effect of the stimulation on the UPDRS, HDRS, and PDSS.

\begin{tabular}{|c|c|c|c|c|c|c|}
\hline & PRE & POST & POST-2 & Factor evaluation & Factor group & Evaluation * Group \\
\hline HDS (score) & $15.833( \pm 5.043)$ & $10.555( \pm 5.113)$ & $10.500( \pm 4.617)$ & $\begin{array}{l}F(2,32)=23.508 \\
p 60.001\end{array}$ & $\begin{array}{l}F(1,16)=0.774 \\
p=0.392\end{array}$ & $\begin{array}{l}F(2,32)=0.389 \\
p=0.681\end{array}$ \\
\hline PDSS (score) & $98.408( \pm 27.746)$ & $112.006( \pm 25.073)$ & $110.813( \pm 23.083)$ & $\begin{array}{l}F(1.412,22.591)=7.902 \\
p=0.005\end{array}$ & $\begin{array}{l}F(1,16)=0.033 \\
p=0.859\end{array}$ & $\begin{array}{l}F(1.412,22.591)=2.932 \\
p=0.089\end{array}$ \\
\hline UPDRS (score) & $50.166( \pm 18.862)$ & $41.944( \pm 19.144)$ & $39.222( \pm 18.405)$ & $\begin{array}{l}F(2,32)=13.077 \\
p 60.001\end{array}$ & $\begin{array}{l}F(1,16)=0.737 \\
p=0.403\end{array}$ & $\begin{array}{l}F(2,32)=0.704 \\
p=0.502\end{array}$ \\
\hline
\end{tabular}

The table represents the effect of factor evaluation (p value) along the whole protocol. Values for each variable are displayed at the beginning of the programme (PRE), after finishing the programme (POST), and one week after POST (POST-2). Values are shown for both groups pooled on the basis that significant interaction Evaluation * Group was never displayed, proving the effect was the same for both groups. Means and standard deviations are displayed, along with the p value.

Abbreviations: HDS, Hamilton Depression Scale; PDSS, Parkinson’s Disease Sleep Scale; UPDRS, Unified Parkinson’s Disease Rating Scale. 
Table 3

Effect of the stimulation on the evaluated parameters of actigraphy.

\begin{tabular}{|c|c|c|c|c|c|c|}
\hline & PRE & POST & POST-2 & Factor evaluation & Factor group & Evaluation * Group \\
\hline Sleep efficiency (\%) & $87.076( \pm 6.232)$ & $85.497( \pm 8.788)$ & $83.210( \pm 11.806)$ & $\begin{array}{l}F(2,32)=1.739 \\
p=0.192\end{array}$ & $\begin{array}{l}F(1,16)=0.099 \\
p=0.757\end{array}$ & $\begin{array}{l}F(2,32)=0.433 \\
p=0.653\end{array}$ \\
\hline Sleep latency (s) & $\begin{array}{l}315.205 \\
( \pm 326.636)\end{array}$ & $\begin{array}{l}502.629 \\
( \pm 514.850)\end{array}$ & $\begin{array}{l}480.240 \\
( \pm 627.189)\end{array}$ & $\begin{array}{l}F(2,32)=1.141 \\
p=0.332\end{array}$ & $\begin{array}{l}F(1,16)=0.893 \\
p=0.359\end{array}$ & $\begin{array}{l}F(2,32)=0.588 \\
p=0.561\end{array}$ \\
\hline Number of sleep bouts & $28.731( \pm 13.725)$ & $28.602( \pm 13.401)$ & $32.184( \pm 20.383)$ & $\begin{array}{l}F(2,32)=1.087 \\
p=0.349\end{array}$ & $\begin{array}{l}F(1,16)=0.646 \\
p=0.433\end{array}$ & $\begin{array}{l}F(2,32)=0.030 \\
p=0.971\end{array}$ \\
\hline Mean Length of sleep bouts (s) & $\begin{array}{l}888.927 \\
( \pm 499.473)\end{array}$ & $\begin{array}{l}1081.001 \\
( \pm 594.033)\end{array}$ & $\begin{array}{l}1156.037 \\
( \pm 942.232)\end{array}$ & $\begin{array}{l}F(2,32)=1.294 \\
p=0.288\end{array}$ & $\begin{array}{l}F(1,16)=0.146 \\
p=0.707\end{array}$ & $\begin{array}{l}F(2,32)=0.162 \\
p=0.851\end{array}$ \\
\hline Mean Length of wake bouts (s) & $83.092( \pm 31.938)$ & $101.593( \pm 42.676)$ & $101.592( \pm 47.672)$ & $\begin{array}{l}F(2,32)=2.608 \\
p=0.089\end{array}$ & $\begin{array}{l}F(1,16)=0.409 \\
p=0.532\end{array}$ & $\begin{array}{l}F(2,32)=0.489 \\
p=0.618\end{array}$ \\
\hline Number of minutes immobile & $\begin{array}{l}384.583 \\
( \pm 88.831)\end{array}$ & $\begin{array}{l}362.745 \\
( \pm 101.171)\end{array}$ & $\begin{array}{l}350.268 \\
( \pm 126.125)\end{array}$ & $\begin{array}{l}F(2,32)=1.130 \\
p=0.336\end{array}$ & $\begin{array}{l}F(1,16)=0.000 \\
p=0.985\end{array}$ & $\begin{array}{l}F(2,32)=0.413 \\
p=0.665\end{array}$ \\
\hline Number of minutes immobile (\%) & $85.232( \pm 6.011)$ & $83.326( \pm 8.590)$ & $77.962( \pm 21.417)$ & $\begin{array}{l}F(1.063,17.002)=1.936 \\
p=0.182\end{array}$ & $\begin{array}{l}F(1,16)=0.990 \\
p=0.334\end{array}$ & $\begin{array}{l}F(1.063,17.002)=0.701 \\
p=0.423\end{array}$ \\
\hline Number of minutes moving & $66.037( \pm 29.234)$ & $72.958( \pm 37.740)$ & $92.888( \pm 78.166)$ & $\begin{array}{l}F(1.120,17.915)=1.996 \\
p=0.175\end{array}$ & $\begin{array}{l}F(1,16)=1.673 \\
p=0.214\end{array}$ & $\begin{array}{l}F(1.120,17.915)=0.584 \\
p=0.473\end{array}$ \\
\hline Number of minutes moving (\%) & $14.767( \pm 6.011)$ & $16.673( \pm 8.590)$ & $22.037( \pm 21.417)$ & $\begin{array}{l}F(1.063,17.002)=1.936 \\
p=0.182\end{array}$ & $\begin{array}{l}F(1,16)=0.990 \\
p=0.334\end{array}$ & $\begin{array}{l}F(1.063,17.002)=0.701 \\
p=0.423\end{array}$ \\
\hline Number of immobile phases & $64.407( \pm 26.656)$ & $68.332( \pm 33.386)$ & $66.453( \pm 40.535)$ & $\begin{array}{l}F(2,32)=0.192 \\
p=0.826\end{array}$ & $\begin{array}{l}F(1,16)=0.139 \\
p=0.715\end{array}$ & $\begin{array}{l}F(2,32)=0.114 \\
p=0.892\end{array}$ \\
\hline Mean length of immobility (min) & $7.248( \pm 3.262)$ & $7.777( \pm 5.855)$ & $7.535( \pm 6.236)$ & $\begin{array}{l}F(2,32)=0.094 \\
p=0.910\end{array}$ & $\begin{array}{l}F(1,16)=0.001 \\
p=0.972\end{array}$ & $\begin{array}{l}F(2,32)=0.848 \\
p=0.438\end{array}$ \\
\hline Immobility phase of 1 min (\#) & $21.889( \pm 13.312)$ & $26.111( \pm 18.872)$ & $24.675( \pm 19.865)$ & $\begin{array}{l}F(2,32)=0.790 \\
p=0.462\end{array}$ & $\begin{array}{l}F(1,16)=0.502 \\
p=0.489\end{array}$ & $\begin{array}{l}F(2,32)=0.062 \\
p=0.940\end{array}$ \\
\hline Immobility of 1 min (\%) & $31.188( \pm 8.062)$ & $32.883( \pm 12.341)$ & $37.246( \pm 19.374)$ & $\begin{array}{l}F(1.422,22.747)=1.539 \\
p=0.235\end{array}$ & $\begin{array}{l}F(1,16)=0.770 \\
p=0.393\end{array}$ & $\begin{array}{l}F(1.422,22.747)=0.542 \\
p=0.530\end{array}$ \\
\hline Movement and fragmentation index & $45.955( \pm 13.677)$ & $49.557( \pm 20.470)$ & $59.284( \pm 40.181)$ & $\begin{array}{l}F(1.179,18.870)=1.826 \\
p=0.194\end{array}$ & $\begin{array}{l}F(1,16)=0.908 \\
p=0.355\end{array}$ & $\begin{array}{l}F(1.179,18.870)=0.649 \\
p=0.455\end{array}$ \\
\hline
\end{tabular}

The table represents the effect of factor evaluation ( $\mathrm{p}$ value) along the whole protocol. Values for each variable are displayed at the beginning of the programme (PRE), after finishing the programme (POST), and one week after POST (POST-2). Values are shown for two groups pooled on the basis that significant interaction Evaluation * Group was never displayed, proving the effect was the same for both groups. Means and week after POST (POST-2). Values are shown for two goviations are displayed, along with the $p$ value. 
index, and sleep efficiency in a group of patients receiving focal parietal stimulation. We were unable to reproduce their results. Most likely the differences accounting for this are methodological; for example, they applied rTMS at $5 \mathrm{~Hz}$, $80 \%$ RMT; used a figure of eight coil over the motor vs. parietal cortex, both bilaterally; and the coil used in our study was a large round diffuse coil, not the same as van Dijk [21] (we did not try to replicate the study since ours was ongoing when van Dijk's was published). The areas of cortical stimulation could also account for different results. Other more minor reasons could be considered (intensity differed for instance). Nevertheless, the main point seems to be that their data, unlike ours, seem not to result completely from a placebo effect; therefore further work is needed. This should not detract from the fact that our data were significantly affected by the placebo response of the patients, under conditions where they subjectively recorded their “improvements." Subjective measures resisted this, paradoxically reflecting both the positive and negative sides of this (and another motor signs [37]) study - patients "quality of life" may be at least partially improved, but this results simply from the expectation that the "treatment" will improve their situation, whereas more objective measures fail to see this improvement and quickly identify the placebo nature of these responses.

\section{Conclusions}

In conclusion, rTMS, under the stimulation parameters used in this study, has no direct therapeutic effect on sleep in PD, but given the scarcity of work assessing the putative therapeutic effect of the technique on Parkinsonian sleep it seems clear further studies are needed. Future studies should control for effects of a placebo response. Also, protocols presented here and by others [21] should be extended to polysomnography.

\section{Conflict of interest}

Nothing to declare.

\section{Acknowledgments}

We thank Dr. J.J. Díaz Silva and the physiotherapists Ms. Ana Novo and Ms. Soraya Núñez for their contribution to this work and Prof. Ricardo Cao for statistical advice. Supported by Xunta de Galicia (Consellería de Educación-2007/000140-0 and Dirección Xeral de I+D+i; PGIDIT06PXIC137004PN), Spain.

\section{References}

[1] Jankovic J. Parkinson’s disease: clinical features and diagnosis. J Neurol Neurosurg Psychiatry 2008;79:368-76.

[2] Askenasy JJ. Sleep disturbances in Parkinsonism. J Neural Transm 2003;110:125-50.

[3] Partinen M. Sleep disorder related to Parkinson's disease. J Neurol 1997;244:S3-6.

[4] Chaudhuri KR, Healy DG, Schapira AHV. Non-motor symptoms of Parkinson's disease: diagnosis and management. Lancet Neurol 2006;5:235-45.

[5] Young A, Horne M, Churchward T, Freezer N, Holmes P, Ho M. Comparison of sleep disturbance in mild versus severe Parkinson's disease. Sleep 2002;25:573-7.

[6] Menza MA, Rosen RC. Sleep in Parkinsons-disease - the role of depression and anxiety. Psychosomatics 1995;36:272-6.

[7] Comella CL. Sleep disorders in Parkinson’s disease: an overview. Mov Disord 2007;22:S367-73.

[8] Tandberg E, Larsen JP, Karlsen K. A community-based study of sleep disorders in patients with Parkinson's disease. Mov Disord 1998;13:895-9.

[9] Comella CL, Morrissey M, Janko K. Nocturnal activity with nighttime pergolide in Parkinson disease: a controlled study using actigraphy. Neurology 2005;64:1450-1.

[10] Chaudhuri KR, Schapira AHV. Non-motor symptoms of Parkinson's disease: dopaminergic pathophysiology and treatment. Lancet Neurol 2009;8:464-74.

[11] Askenasy JJM, Yahr MD. Reversal of sleep disturbance in Parkinsons-disease by antiparkinsonian therapy - a preliminary-study. Neurology 1985;35:527-32.

[12] Stocchi F, Barbato L, Nordera G, Berardelli A, Ruggieri S. Sleep disorders in Parkinson’s disease. J Neurol 1998;245:S15-8.

[13] Hallett M. Transcranial magnetic stimulation: a primer. Neuron 2007;55:187-99.

[14] Wassermann EM, Lisanby SH. Therapeutic application of repetitive transcranial magnetic stimulation: a review. Clin Neurophysiol 2001;112:1367-77.

[15] Edwards MJ, Talelli P, Rothwell JC. Clinical applications of transcranial magnetic stimulation in patients with movement disorders. Lancet Neurol 2008;7:827-40.

[16] Valero-Cabré A, Payne BR, Pascual-Leone A. Opposite impact on (14)C-2-deoxyglucose brain metabolism following patterns of high and low frequency repetitive transcranial magnetic stimulation in the posterior parietal cortex. Exp Brain Res 2007;176:603-15.

[17] Di Lazzaro V, Oliviero A, Pilato F, Saturno E, Insola A, Mazzone P, et al. Descending volleys evoked by transcranial magnetic stimulation of the brain in conscious humans: effects of coil shape. Clin Neurophysiol 2002;113(1):114-9.

[18] Di Lazzaro V, Oliviero A, Mazzone P, Insola A, Pilato F, Saturno E, et al. Comparison of descending volleys evoked by monophasic and biphasic magnetic stimulation of the motor cortex in conscious humans. Exp Brain Res 2001;141:121-7. 
[19] Sommer M, Alfaro A, Rummel M, Speck S, Lang N, Tings T, et al. Half sine, monophasic and biphasic transcranial magnetic stimulation of the human motor cortex. Clin Neurophysiol 2006;117(4):838-44.

[20] Di Lazzaro V, Oliviero A, Saturno E, Pilato F, Insola A, Mazzone P, et al. The effect on corticospinal volleys of reversing the direction of current induced in the motor cortex by transcranial magnetic stimulation. Exp Brain Res 2001;138(2):268-73.

[21] van Dijk KD, Most EIS, van Someren EJW, Berendse HW, van der Werf YD. Beneficial effect of transcranial magnetic stimulation on sleep in Parkinson's disease. Mov Disord 2009;24:878-84.

[22] Huber R, Esser SK, Ferrarelli F, Massimini M, Peterson MJ, Tononi G. TMSinduced cortical potentiation during wakefulness locally increases slow wave activity during sleep. PLoS One 2007;2(3):e276.

[23] Brunner H, Wetter TC, Hogl B, Yassouridis A, Trenkwalder C, Friess E. Microstructure of the non-rapid eye movement sleep electroencephalogram in patients with newly diagnosed Parkinson's disease: effects of dopaminergic treatment. Mov Disord 2002;17:928-33.

[24] Massimini M, Ferrarelli F, Esser SK, Riedner BA, Huber R, Murphy M, et al. Triggering sleep slow waves by transcranial magnetic stimulation. Proc Natl Acad Sci USA 2007;104(20):8496-501.

[25] Mally J, Stone TW. Improvement in Parkinsonian symptoms after repetitive transcranial magnetic stimulation. J Neurol Sci 1999;162(2):179-84.

[26] Mally J, Stone TW. Therapeutic and “dose-dependent” effect of repetitive microelectroshock induced by transcranial magnetic stimulation in Parkinson’s disease. J Neurosci Res 1999;57(6):935-40.

[27] Lomarev MP, Kanchana S, Bara-Jimenez W, Iyer M, Wassermann EM, Hallett M. Placebo-controlled study of rTMS for the treatment of Parkinson's disease. Mov Disord 2006;21:325-31.

[28] Sadeh A, Hauri PJ, Kripke DF, Lavie P. The role of actigraphy in the evaluation of sleep disorders. Sleep 1995;18:288-302.

[29] Kushida CA, Chang A, Gadkary C, Guilleminault C, Carrillo O, Dement WC. Comparison of actigraphic, polysomnographic, and subjective assessment of sleep parameters in sleep-disordered patients. Sleep Med 2001;2:389-96.

[30] van Hilten B, Hoff JI, Middelkoop HA, van der Velde EA, Kerkhof GA, Wauquier A, et al. Sleep disruption in Parkinson's disease. Assessment by continuous activity monitoring. Arch Neurol 1994;51:922-8.

[31] Chaudhuri KR, Pal S, DiMarco A, Whately-Smith C, Bridgman K, Mathew R, et al. The Parkinson's disease sleep scale: a new instrument for assessing sleep and nocturnal disability in Parkinson’s disease. J Neurol Neurosurg Psychiatry 2002;73:629-35.

[32] Chaudhuri KR, Martinez-Martin P. Clinical assessment of nocturnal disability in Parkinson's disease - the Parkinson's disease sleep scale. Neurology 2004;63:S17-20.

[33] Martinez-Martin P, Salvador C, Menéndez-Guisasola L, González S, Tobías A, Almazán J, et al. Parkinson’s disease sleep scale: validation study of a Spanish version. Mov Disord 2004;19:1227-32.

[34] Perez-Lloret S, Rossi M, Nouzeilles MI, Trenkwalder C, Cardinali DP, Merello M. Parkinson’s disease sleep scale, sleep logs, and actigraphy in the evaluation of sleep in Parkinsonian patients. J Neurol 2009;256:1480-4.

[35] Hamilton M. A rating scale for depression. J Neurol Neurosurg Psychiatry 1960;23:56-62.

[36] Ramos-Brieva JA, Cordero-Villafafila A. A new validation of the Hamilton rating scale for depression. J Psychiatr Res 1988;22:21-8.

[37] Okabe S, Ugawa Y, Kanazawa I. Effectiveness of rTMS on Parkinson's disease study group. 0.2-Hz repetitive transcranial magnetic stimulation has no addon effects as compared to a realistic sham stimulation in Parkinson's disease. Mov Disord 2003;18:382-8. 